\title{
Elevated serum CA72-4 levels predict poor prognosis in pancreatic adenocarcinoma after intensity-modulated radiation therapy
}

\author{
Peng Liu ${ }^{1}$, Yuan Zhu ${ }^{1}$ and Luying Liu ${ }^{1}$ \\ ${ }^{1}$ Department of Radiotherapy, Zhejiang Cancer Hospital, Hangzhou, Zhejiang, China \\ Correspondence to: Peng Liu, email: sololiup@126.com \\ Keywords: Pancreatic adenocarcinoma, CA72-4, Prognosis, Radiotherapy \\ Received: January 06, $2015 \quad$ Accepted: February 10, $2015 \quad$ Published: March 12, 2015 \\ This is an open-access article distributed under the terms of the Creative Commons Attribution License, which permits unrestricted use, \\ distribution, and reproduction in any medium, provided the original author and source are credited.
}

\section{ABSTRACT}

Carbohydrate antigen 72-4 (CA72-4) is a human tumor-associated glycoprotein, commonly used as a tumor marker for diagnosing and predicting outcome in gastric and ovarian cancers. However, the relationship between serum CA72-4 levels and prognosis of pancreatic adenocarcinoma has not been fully elucidated. A total of 113 consecutive locally advanced pancreatic adenocarcinoma patients who underwent intensity-modulated radiation therapy (IMRT) with or without chemotherapy were enrolled in this study. Serum CA72-4 levels were analyzed using immunoenzymometric assays. The association between serum CA72-4 levels and prognosis was evaluated. Serum CA72-4 levels was related with lymph node metastasis $(P<0.001)$. The median overall survival time was 14.0 months for patients with serum CA72-4 normal levels and 10.0 months for the elevated levels $(P<0.001)$. Multivariate analysis identified that Serum CA72-4 concentration was a significant prognostic factor $(P<0.001)$. The hazard ratio (HR) of elevated serum CA72-4 levels compared with normal serum CA72-4 levels was 2.34 (95\% confidence interval [CI]: 1.46-3.73), after adjusted for gender and age. Based on this finding, Serum CA72-4 is a potential marker to predict lymph node metastasis and prognosis in pancreatic adenocarcinoma.

\section{INTRODUCTION}

Pancreatic adenocarcinoma is one of the most extremely malignant neoplasms in both developing and developed countries. The incidence has been increasing in recent years, recorded rate of 8 per 100 000. A great number of pancreatic adenocarcinoma patients experienced disease progression in a very short time. Radical surgery with adjuvant chemotherapy is thought to be the single modality that can provide opportunity of cure and long-time survival [1]. However, due to poor early diagnosis, only $13-20 \%$ of patients are at stage $I$ at the time of diagnosis. For locally advanced pancreatic adenocarcinoma, combined or sequential chemotherapy and radiotherapy is the standard method $[2,3]$. Chemoradiation therapy displays a modest survival benefit compared with radiotherapy or chemotherapy alone $[4,5]$. However, some patients with similar clinical stage have remarkably different survival prognosis. In this way, heterogeneity of protein expression profiles may play a very important role in the development of pancreatic adenocarcinoma [6]. Various molecular biomarkers are useful to identify the biological characteristics and predict the behavior of pancreatic adenocarcinoma. However, to date, most of these markers had not been proven to be sufficiently effective [7].

Carbohydrate antigen 19-9 (CA19-9) is considered to be the best prognostic serum marker for pancreatic cancer [8]. Increased baseline CA19-9 is related with low resectability, early metastasis and short survival time. However, the clinical utility of CA19-9 to identify the patients who are at high risk for disease progression is still limited [9]. Carbohydrate antigen 72-4 (CA72-4) is a human tumor-associated glycoprotein of high molecular weight, detected by monoclonal antibody $\mathrm{CC} 49$ and B72.3. CA72-4 is commonly used as a tumor marker for diagnosing and predicting outcome in gastric [10] and ovarian cancers [11]. CA72-4 is shown to provide independent prognostic information in pancreatic cancer postoperation. In Louhimo J et al's study [12], CA72- 
Table 1: Relationship between serum CA72-4 levels and clinicopathological variables $(\mathbf{n}=113)$

\begin{tabular}{|c|c|c|c|c|}
\hline & & \multicolumn{2}{|c|}{ serum CA72-4 levels } & \multirow[t]{2}{*}{$\mathbf{P}$} \\
\hline Variables & Total (n) & CA $72-4 \leq 6.9 \mathrm{U} / \mathrm{mL}(\mathrm{n}=71)$ & CA $72-4>6.9 \mathrm{U} / \mathrm{mL}(\mathrm{n}=42)$ & \\
\hline Gender & & & & \\
\hline Male & 71 & $41(57.7 \%)$ & $30(42.3 \%)$ & 0.146 \\
\hline Female & 42 & $30(71.4 \%)$ & $12(28.6 \%)$ & \\
\hline Age & & & & \\
\hline$<65$ & 80 & $52(65.0 \%)$ & $28(35.0 \%)$ & 0.458 \\
\hline$\geq 65$ & 33 & $19(57.6 \%)$ & $14(42.4 \%)$ & \\
\hline Smoking status & & & & \\
\hline Never & 58 & $44(75.9 \%)$ & $14(24.1 \%)$ & 0.003 \\
\hline Current/ever & 55 & $27(49.1 \%)$ & $28(50.9 \%)$ & \\
\hline Tumor location & & & & \\
\hline Head & 57 & $35(61.4 \%)$ & $22(38.6 \%)$ & 0.322 \\
\hline Body and tail & 56 & $36(64.3 \%)$ & $20(35.7 \%)$ & \\
\hline T stage & & & & \\
\hline $\mathrm{T}_{1-2}$ & 29 & $16(55.2 \%)$ & $13(44.8 \%)$ & 0.322 \\
\hline $\mathrm{T}_{3-4}$ & 84 & $55(65.5 \%)$ & $29(34.5 \%)$ & \\
\hline $\mathrm{N}$ stage & & & & \\
\hline $\mathrm{N}_{0}$ & 60 & $47(78.3 \%)$ & $13(21.7 \%)$ & $<0.001$ \\
\hline $\mathrm{N}_{1}$ & 53 & $24(45.3 \%)$ & $29(54.7 \%)$ & \\
\hline Treatment Response & & & & \\
\hline $\mathrm{CR}+\mathrm{PR}$ & 37 & $27(73.0 \%)$ & $10(27.0 \%)$ & 0.120 \\
\hline $\mathrm{SD}+\mathrm{PD}$ & 76 & $44(57.6 \%)$ & $32(42.1 \%)$ & \\
\hline
\end{tabular}


4 is superior to CA19-9 in predicting the outcome of pancreatic cancer. To our knowledge, no report has been published regarding the relationship between serum CA72-4 levels and clinicopathological features and prognosis of elocally advanced pancreatic adenocarcinoma treated with intensity-modulated radiation therapy (IMRT). Therefore, the objectives of this study were (1) to examine serum CA72-4 levels in 113 locally advanced pancreatic adenocarcinoma, (2) to assess the association between serum CA72-4 levels and clinicopathological features in locally advanced pancreatic adenocarcinoma, (3) to evaluate the prognostic impact of serum CA72-4 levels on overall survival in patients with locally advanced pancreatic adenocarcinoma.

\section{RESULTS}

This survival study was carried out in 113 consecutive locally advanced pancreatic adenocarcinoma patients. The baseline characteristics of these patients are summarized in Table 1. A total of 71 men and 42 women were included with ages ranging from 34 to 78 years (median, 60 years). Primary tumor site was head in 57 patients, body and tail in 56 patients. There were 14 $(12.4 \%) \mathrm{T}_{1}, 15(13.3 \%) \mathrm{T}_{2}, 47(41.6 \%) \mathrm{T}_{3}$, and $37(32.7 \%)$ $\mathrm{T}_{4}$. In terms of the new AJCC TNM staging system, 60 patients $(53.1 \%)$ were categorized as $\mathrm{N}_{0}$ and $53(46.9 \%)$ as $\mathrm{N}_{1}$. All the patients were included in the survival analysis. The overall follow-up durations ranged from 3 to 45 months (median, 12.0 months). 94 of 113 patients died of disease progression.

Seven patients $(6.2 \%)$ achieved a complete response (CR) as the best response post-treatment. The median overall survival time was 36 months. Thirty patients $(26.5 \%, 14$ in head of pancreas and 16 in body and tail of pancreas) had a partial response (PR) with median overall survival time 15.5 months. The clinical benefit rate, calculated as CR+PR, was $32.7 \%$. 68 patients $(60.2 \%)$ had stable disease (SD). The median overall survival time was 8 months. Progressive disease (PD) was observed in 8 patients $(7.1 \%)$. The median overall survival time was only 7 months.

\section{Relationship between serum CA72-4 levels and clinicopathological parameters}

The relationship between serum CA72-4 levels and clinicopathological parameters is presented in the Table1. The median of serum CA72-4 levels in all patients was 4.05 U/mL (range: 0.10-173.61 U/mL). Forty-two patients (37.2\%) had serum CA72-4 levels more than $6.9 \mathrm{U} / \mathrm{mL}$, defined as elevated group according to the manufacture's instruction. A cut-off point of 65 years was established to determine whether age affects serum CA72-4 levels and overall survival. There was no relationship between serum
CA72-4 levels and patients' age ( $\mathrm{P}>0.05)$. Serum CA72-4 levels were significantly higher in males than in females (mean, 11.9 U/mL Vs 10.4 U/mL, $\mathrm{P}<0.05$ ). The Chi-square test also exhibited that there was near to be significant
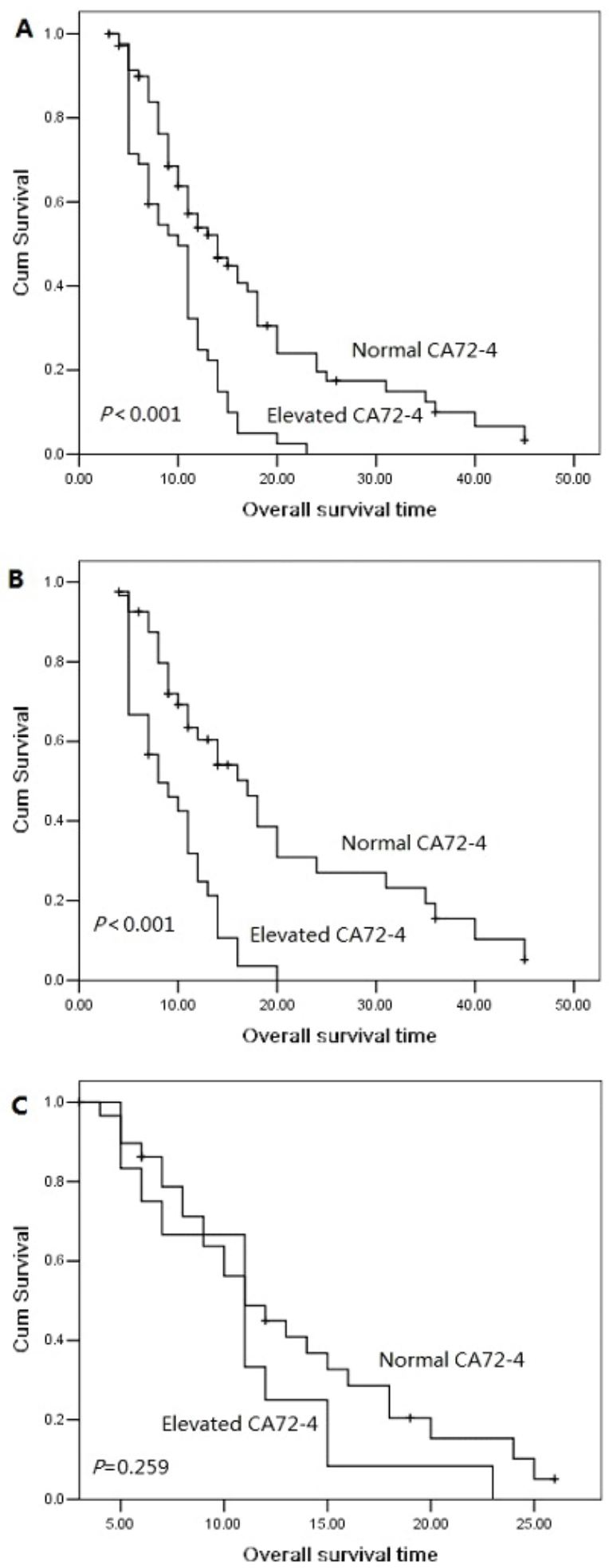

Figure 1: Overall survival curves with serum CA72-4 levels in the entire population (A), in female patients (B) and in male patients (C). 
Table 2: Univariable analysis for the effect of serum CA 72-4 levels on overall survival (n=113)

\begin{tabular}{|c|c|c|c|}
\hline \multirow{2}{*}{ Variables } & \multicolumn{3}{|c|}{ Univariable analysis } \\
\hline & HR & $95 \% \mathrm{CI}$ & $\mathbf{P}$ \\
\hline Gender (Male Vs Female) & 0.84 & $0.55-1.29$ & 0.424 \\
\hline Age $(<65 \mathrm{Vs} \geq 65)$ & 0.93 & $0.59-1.45$ & 0.740 \\
\hline Tumor location (Head Vs Body and tail) & 0.78 & $0.52-1.18$ & 0.242 \\
\hline T stage $\left(\mathrm{T}_{1-2} \mathrm{Vs} \mathrm{T}_{3-4}\right)$ & 0.97 & $0.61-1.54$ & 0.897 \\
\hline $\mathrm{N}$ stage $\left(\mathrm{N}_{0} \mathrm{Vs} \mathrm{N}_{1}\right)$ & 0.99 & $0.66-1.48$ & 0.942 \\
\hline Smoking status (Current, ever Vs Never) & 1.98 & $1.29-3,03$ & 0.002 \\
\hline Chemotherapy (Yes Vs No) & 0.72 & $0.48-1.09$ & 0.125 \\
\hline Treatment response (CR+PR Vs SD+PD) & 4.81 & $2.87-8.05$ & $<0.001$ \\
\hline $\begin{array}{c}\text { CA 72-4 levels (CA 72-4>6.9 U/mL Vs CA } \\
72-4 \leq 6.9 \mathrm{U} / \mathrm{mL})\end{array}$ & 2.36 & $1.53-3.65$ & $<0.001$ \\
\hline
\end{tabular}

Table 3: Multivariable analysis for serum CA 72-4 levels on overall survival (n=113)

\begin{tabular}{|c|c|c|c|}
\hline \multirow{2}{*}{ Variables } & \multicolumn{3}{|c|}{ Multivariable analysis } \\
\hline & HR & $95 \% \mathrm{CI}$ & $\mathbf{P}$ \\
\hline Smoking status (Current, ever Vs Never) & 1.26 & $0.81-1.97$ & 0.297 \\
\hline $\begin{array}{c}\text { CA 72-4 levels (CA 72-4>6.9 U/mL Vs CA } \\
72-4 \leq 6.9 \mathrm{U} / \mathrm{mL}) \\
\end{array}$ & 1.97 & $1.27-3.08$ & 0.003 \\
\hline Treatment response & 4.30 & $2.55-7.27$ & $<0.001$ \\
\hline After adjusted for gender and age & & & \\
\hline Smoking status (Current, ever Vs Never) & 1.25 & $0.80-1.95$ & 0.332 \\
\hline $\begin{array}{c}\text { CA } 72-4 \text { levels (CA } 72-4>6.9 \mathrm{U} / \mathrm{mL} \text { Vs CA } \\
72-4 \leq 6.9 \mathrm{U} / \mathrm{mL}) \\
\end{array}$ & 2.34 & $1.46-3.73$ & $<0.001$ \\
\hline Treatment response & 5.52 & $3.17-9.62$ & $<0.001$ \\
\hline
\end{tabular}
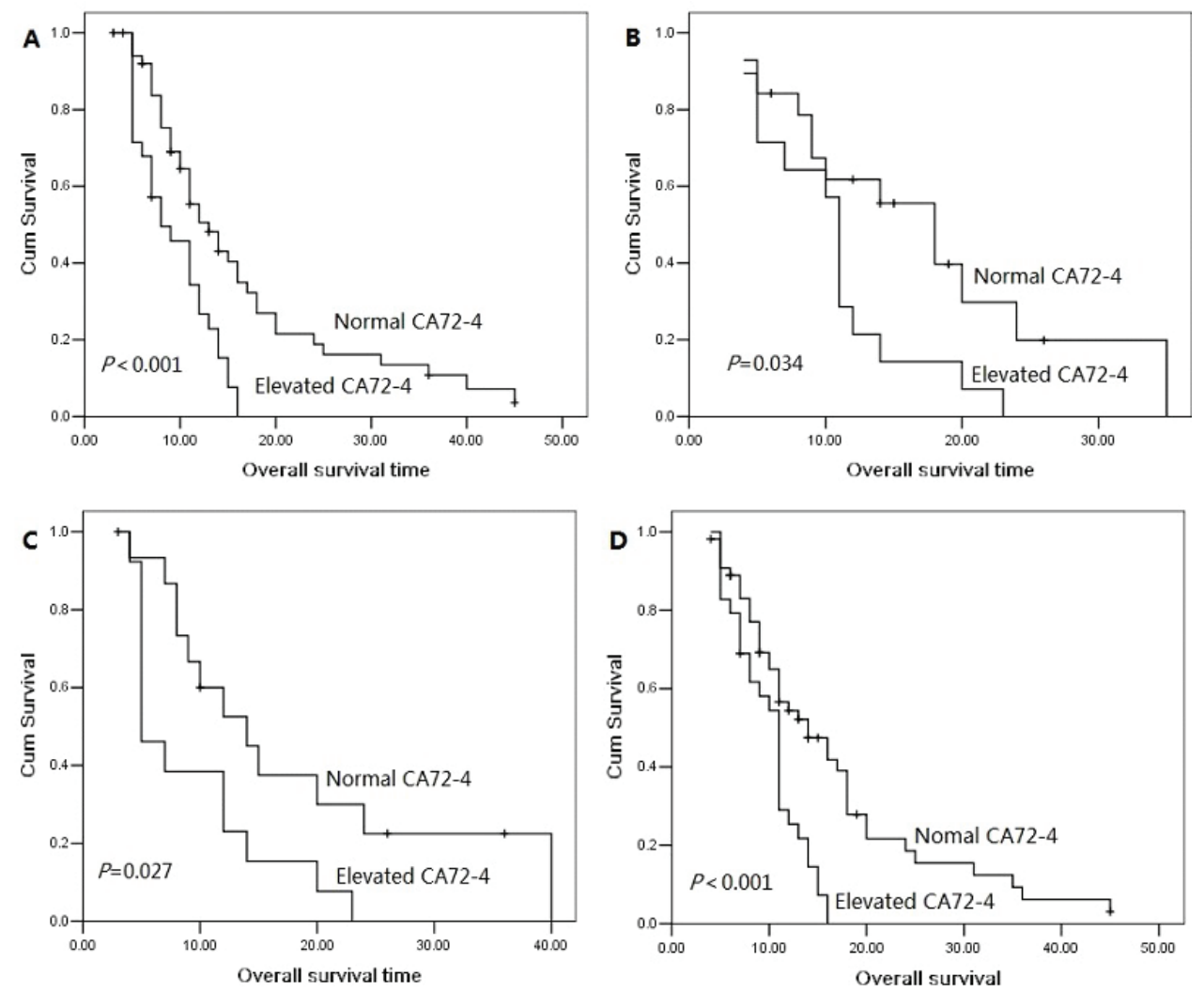

Figure 2: Overall survival curves with serum CA72-4 levels in age $<65$ patients (A), age $\geq 65$ patients (B), stage T1-2 patients (C) and stage T3-4 patient (D). 
difference between male patients and female patients $(\mathrm{P}=0.146)$. Serum CA72-4 levels were also related with $\mathrm{N}$ stage $(\mathrm{P}<0.001)$. There was no significant correlation between serum CA72- levels and tumor location and $\mathrm{T}$ stage $(\mathrm{P}>0.05)$. Serum CA72-4 levels in smoking pancreatic adenocarcinoma patients were significantly higher than never smoking patients (mean: $13.15 \mathrm{U} / \mathrm{mL}$ Vs $2.46 \mathrm{U} / \mathrm{mL}, \mathrm{P}=0.004)$. Serum CA72-4 levels did not relate with treatment response $(\mathrm{P}=0.120)$, even though circulating CA72-4 levels were slightly higher in patients with $\mathrm{CR}+\mathrm{PR}$ than those with $\mathrm{SD}+\mathrm{PD}$ (t-test, $\mathrm{P}=0.136$ ).

\section{Serum CA72-4 levels and patients' prognosis with univariable and multivariable analysis}

The 1 year, 2 year and 3 year cumulative overall survival (OS) rates were $42.4 \%, 13.8 \%$ and $5.7 \%$, respectively. The median overall survival time was 14.0 months for patients with serum CA72-4 normal levels and 10.0 months for elevated levels. The 2 year overall survival rate was $19.6 \%$ for patients with serum CA724 normal levels and $0 \%$ for elevated levels, indicating a significantly worse prognosis in serum CA72-4 elevated levels ( $\mathrm{P}<0.001$, Figure $1 \mathrm{~A})$. The median overall survival time was shorter in patients with serum CA72-4 elevated levels than in those with normal levels in male pancreatic adenocarcinoma patients ( 8 months Vs 17 months, $\mathrm{P}<0.001$, Figure 1B). However, in the population of female patients, there was no significant difference of median OS between those with serum CA72-4 normal levels (median $\mathrm{OS}=11$ months) and those with serum CA72-4 elevated levels (median $\mathrm{OS}=11$ months) $(\mathrm{P}=0.259)$ (Figure 1C). Two-year survival probability was $0 \%$ for serum CA72-4 elevated levels patients versus $18.8 \%$ for serum CA72-4 normal levels patients with age $<65$ years $(\mathrm{P}=0.004$, Figure 2A). Similarly, patients with serum CA72-4 elevated levels had shorter OS than those with serum CA72-4 normal levels in the patients with age $\geq 65$ years (median OS: 11.0 months Vs 18.0 months, $\mathrm{P}=0.034$, Figure $2 \mathrm{~B}$ ). There was also significant difference of OS between serum CA72-4 normal levels patients and serum CA72-4 elevated levels patients among stage $\mathrm{T}_{1-2}$ (Median OS: 14.0 months Vs 5.0months, $\mathrm{P}=0.027$, Figure $2 \mathrm{C}$ ) and stage $\mathrm{T}_{3-4}$ (Median OS: 14.0 months Vs 11.0 months, $\mathrm{P}<0.001$, Figure 2D). Furthermore, we examined the overall survival differences of patients stratified for normal CA72-4 levels and elevated CA72-4 levels according to regional lymph node metastasis. We found that for patients without lymph node metastasis, a highly significantly inferior outcome was observed in patients with elevated serum CA72-4 levels, compared with normal serum CA72-4 levels (median OS: 8 months Vs 12 months, $\mathrm{P}=0.001$, Figure 3A). Also, there was significant difference in OS between patients with elevated serum CA72-4 levels and normal serum CA72-4 levels (median OS: 11 months Vs 17 months, $\mathrm{P}=0.001$, Figure $3 \mathrm{~B}$ ) in the patients with positive lymph node metastasis. Elevated serum CA72-4 levels were also significantly associated with shorter OS for patients with chemotherapy $(\mathrm{P}=0.007$, Figure $3 \mathrm{C})$ and patients without chemotherapy $(P=0.007$, Figure 3D).

We performed univariate analysis for serum CA72-4 levels and other 8 clinicopathological variables (including gender, age, tumor location, T stage, lymph node metastasis, smoking status, treatment response and chemotherapy) to find useful prognostic factors for pancreatic adenocarcinoma. Serum CA72-4 levels, treatment response and smoking status were three significant prognostic factors (serum CA72-4 levels: $\mathrm{P}<0.001$; treatment response: $\mathrm{P}<0.001$; smoking status: $\mathrm{P}=0.002$ ). The hazard ratio (HR) of elevated serum CA72-4 levels compared with normal serum CA72-4 levels was 2.36 (95\% confidence interval [CI]: 1.53-3.65), indicating that elevated serum CA72-4 levels increased 2.36-fold higher risk for cancer-related death. We then performed multivariate analysis for these factors whose presence significantly affected prognosis. Patients with elevated serum CA72-4 levels had a 1.97-fold increased risk of death compared to those with normal serum CA724 levels. The 95\% CI was 1.27-3.08. When adjusted for patients' gender, age, the adjusted HR of elevated serum CA72-4 levels became 2.34 (95\% CI: 1.46-3.73) in comparison with normal serum CA72-4 levels. These finding indicated that pretreatment serum CA72-4 is a potential marker to predict lymph node metastasis and prognosis in pancreatic adenocarcinoma.

\section{DISCUSSION}

In this study, we find that elevated pre-treatment serum CA72-4 levels are associated with poorer prognosis in pancreatic adenocarcinoma patients receiving IMRT. Serum CA72-4 levels were significantly higher in smoking patients $(\mathrm{P}=0.003)$. Patients with elevated serum CA724 levels had 2.34 times the risk of death compared with those with normal serum CA72-4 levels adjusted for patients' gender, age. Furthermore, our study demonstrated that serum CA72-4 levels were significantly related with cancer cell lymph node metastasis, in agreement with previous reports in gastric cancer [13]. Commonly, lymphatic metastasis is an early event in pancreatic adenocarcinoma, which is a strong prognostic factor for pancreatic adenocarcinoma. These result indicated that CA72-4 may be involved in the process of pancreatic adenocarcinoma from local disease to systematic disease, which subsequently results in inferior outcome.

Jiang et al [14] found that serum CA72-4 was relatively higher in pancreatic cancer patients but lower in patients with pancreatic benign disease. Testing CA724 combined with CA19-9 may increase the sensitivity and specificity in the diagnosis of pancreatic cancer. Moreover, after radical pancreatic mass resection, serum CA72-4 levels would be decrease significantly. In a recent 
report [15], the specificity of diagnosis of the combined detection of CA19-9 and CA72-4 was increased to $92.8 \%$. In Louhimo et al's study [12], preoperative serum CA724 concentration was an independent novel prognostic biomarker in operable pancreatic cancer. However, the study included both pancreatic adenocarcinoma and endocrine malignancy. In our study, serum CA72-4 levels were observed elevated in $37.2 \%$ patients $(42 / 113)$, and most of them were male patients. Current/ever smoking status may be related with higher serum CA72-4 levels. The univariated and multivariated analysis revealed that serum CA72-4 levels were significantly related with patients' outcome receiving IMRT, with chemotherapy or without chemotherapy.

There are growing evidences to support the theory that CA72-4 plays important role in tumor cell survivorship and progression [16]. However, the exact reason between elevated serum CA72-4 levels and worse prognosis of pancreatic adenocarcinoma remains unknown. CA72-4 is rarely expressed in the most benign tumors and normal adult tissues. Overexpression of CA72-4 could be related with cancer incidents. Szekanecz et al [17] reported that CA72-4 may serve as cell adhesion molecules. Cell adhesion is considered to be a critical process in the migration and metastasis of pancreatic cancer. CA72-
4, like other tumor antigens CA19-9 and CA125, may mediate tumor cell adhesion [18], involve in the metastasis and influence the prognosis of pancreatic adenocarcinoma patients. In our study, CA72-4 is associated with regional lymph node metastasis, revealing that CA72-4 may play a vital part in the tumor cell metastasis. Therefore, CA724 is involved in the regulation mechanism of tumor cell adhesion and its specific role in tumor cell metastasis is worthy of being further study.

The major limitation in this study is that the information on post-treatment local recurrence or metastasis was insufficient. One of the least convincing things in this study is lack the data of disease free survival, although overall survival is the standard indicator in the cancer prognosis study. Second, this retrospective study is relatively small sample size. A prospective study is required to determine the prognostic value of serum CA72-4 levels. Furthermore, this study used traditional method to determine the concentration of serum CA72-4, more sensitive and specific techniques, like real time RTPCR or ultrasensitive electrochemical immunosensor [19] are needed.

In summary, the present study revealed that elevated serum CA72-4 levels are significantly related with metastasis and progression of pancreatic adenocarcinoma.
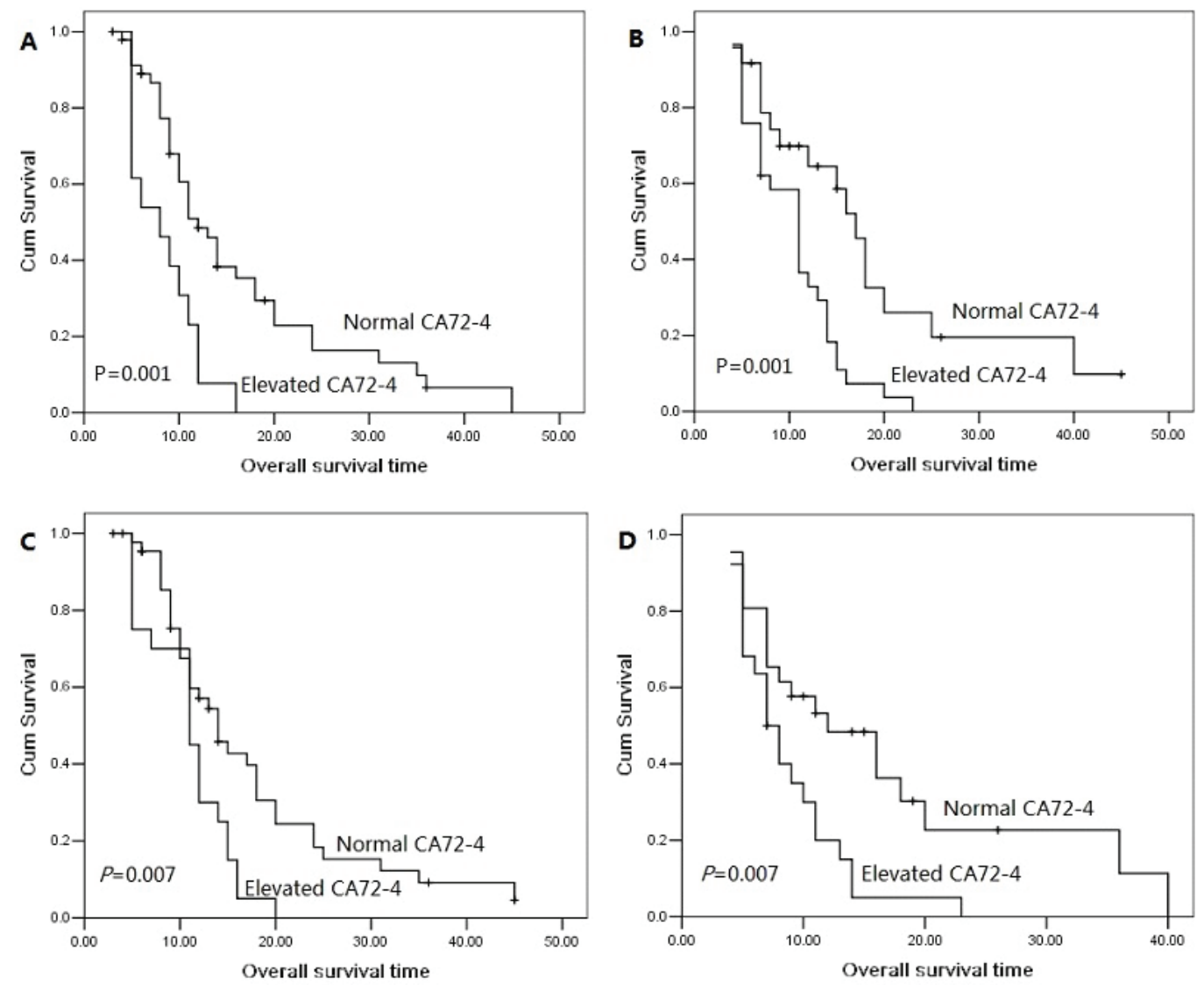

Figure 3: Overall survival curves with serum CA72-4 levels in stage N0 patients (A), in stage N1 patients (B), in patients with chemotherapy $(C)$, in patients without chemotherapy (D). 
Pre-treatment serum CA72-4 levels are new novel independent prognostic biomarker for overall survival in pancreatic adenocarcinma receiving IMRT with or without chemotherapy. This finding provides information of further probe into the biological relevance of CA72-4 in pancreatic adenocarcinoma.

\section{MATERIALS AND METHODS}

A total of 122 consecutive locally advanced pancreatic adenocarcinoma patients who underwent IMRT with or without chemotherapy from January 2010 to December 2013 at Zhejiang Cancer Hospital. All patients were newly confirmed to have pancreatic adenocarcinoma and had not received treatment previously. Patients with other malignancies were excluded from this study. Finally, a total of 113 eligible patients enrolled in our study. Each case was reassigned for tumor, node and metastases (TNM stage) classification and clinical stage according to the American Joint Committee on Cancer (AJCC) staging system [20]. The following detail clinical information was retrospectively collected and analyzed for each case: gender, age at treatment, smoking status, tumor location, clinical TNM stage, treatment response and overall survival (OS) after IMRT. Our study was approved by the institutional review board of the hospital. All patients provided informed consent before IMRT. Overall survival was calculated as the time from radiotherapy to death or censoring.

Each patient provided 5-mL blood sample pretreatment. The drawn blood samples were stored at $-20^{\circ} \mathrm{C}$ until analysis. Serum CA72-4 levels were analyzed using immunoenzymometric assays (Immuno 1, Bayer, Tarrytown, NY). The recommended cut-off value for diagnostic purpose was $6.9 \mathrm{U} / \mathrm{mL}$ for CA72-4.

\section{Treatment schedule}

All patients were immobilized in a supine position, arms overhead, with thermoplastic cast. Intravenous and oral contrast-contrast CT-simulation was performed at $5 \mathrm{~mm}$ intervals of abdomen using CT simulator (GE, Lightspeed, USA). Radiation plans for IMRT were generated using Pinnacle Version 8.0. All patients underwent external beam radiation therapy with $6 \mathrm{MV}$ $\mathrm{X}$-rays. The area of solid macroscopic tumors in pancreas, the surrounding tissue infiltrated and the regional lymph node metastasis were defined as the gross tumor volume (GTV). The GTV plus a margin of at least $5 \mathrm{~mm}$, including any areas of microscopic spread and the regional lymph nodes (peripancreatic, celiac, superior mesenteric, portal hepatic, retroperitoneal), was defied as the clinical target volume (CTV). The plan target volume (PTV) was defined as the CTV plus $0.5-1.0 \mathrm{~cm}$ to account for the daily setup variation and respiratory movement. A fractional daily dose of 1.8 Gy (5 days per week, over 5 weeks) at an isocenter was prescribed. The median delivered dose of IMRT was 50 Gy (ranged: 44.0-55.8Gy). The dose to the adjacent normal structures was constrained as follows: The liver dose was limited to $\mathrm{V}_{5}<75 \%, \mathrm{~V}_{20}<50 \%, \mathrm{~V}_{30}<30 \%$ and a mean dose less than $28 \mathrm{~Gy}$. The kidney dose was limited to $\mathrm{V}_{12}<50 \%$ and $\mathrm{V}_{22.5}<30 \%$. Spinal cord maximum dose was held to $45 \mathrm{~Gy}$. The PTV encompassed at least 95\% isodose line. The dose volume histogram (DVH) was obtained for CTV, PTV, spinal cord, liver and kidney. The small bowel contour was confined to the small bowel loops within $3 \mathrm{~cm}$ of the PTV and was limited to maximal dose $<54$ Gy. Sixty-five patients received two cycles of gemcitabine or S-1 based concurrent chemotherapy. S-1 was administered at $50 \mathrm{mg} / \mathrm{m}^{2}$ twice daily from day 1 to day 14, and gemcitabine was administered by 30-min intravenous infusions of $1000 \mathrm{mg} / \mathrm{m}^{2}$ on day 1 and day 8 of a 21-d cycle.

The CR (complete response), PR (partial response), SD (stable disease) and PD (progressive disease) were assessed at an interval of at least 4 weeks to confirm the objective response. All patients received standardized follow-up, occurring at 3 months interval for two years, 6 months interval the third year, and yearly thereafter. Evaluations comprised a physical examination, complete blood count, liver and kidney function tests, abdominal ultrasound or CT, chest radiography and pelvic CT.

\section{Statistical analysis}

A post-treatment follow-up of the patients was carried out in order to evaluate the impact of CA72-4 on the overall survival. Overall survival was defined as the time interval from the initial event (radiotherapy) to the death or censoring. Serum CA72-4 levels was analyzed as a continuous variable and a categorical variable after grouping by normal levels $(\leq 6.9 \mathrm{U} / \mathrm{mL})$ and elevated levels $(>6.9 \mathrm{U} / \mathrm{mL})$. The chi-square test was performed to evaluate the association between the clinicopathological variables and serum CA72-4 levels. Unpaired student's t-test was used to compare serum CA72-4 levels according to clinicopathological variables. Survival curves were estimated by the univariate Kaplan-Meier method. The log-rank test was applied to check the significant differences in the curves among groups. Furthermore, we used the Cox proportional hazards model with the backward selection method for multivariate analysis. All statistical calculations were performed with SPSS 13.0 for Windows (Chicago, IL). Two-sides P values of $<0.05$ were considered statistical significance.

\section{CONFLICT OF INTEREST}

The authors declare no conflict of interest. 


\section{REFERENCES}

1. Klinkenbijl JH, Jeekel J, Sahmoud T, van Pel R, Couvreur ML, Veenhof CH, Arnaud JP, Gonzalez DG, de Wit LT, Hennipman A and Wils J. Adjuvant radiotherapy and 5-fluorouracil after curative resection of cancer of the pancreas and periampullary region: phase III trial of the EORTC gastrointestinal tract cancer cooperative group. Ann Surg. 1999; 230(6): 776-782; discussion 782-784.

2. Huguet F, Girard N, Guerche CS, Hennequin C, Mornex $\mathrm{F}$ and Azria D. Chemoradiotherapy in the management of locally advanced pancreatic carcinoma: a qualitative systematic review. J Clin Oncol. 2009; 27(13): 2269-2277.

3. Blackstock AW, Tepper JE, Niedwiecki D, Hollis DR, Mayer RJ and Tempero MA. Cancer and leukemia group B (CALGB) 89805: phase II chemoradiation trial using gemcitabine in patients with locoregional adenocarcinoma of the pancreas. Int J Gastrointest Cancer. 2003; 34(2-3): 107-116.

4. Murphy JD, Adusumilli S, Griffith KA, Ray ME, Zalupski MM, Lawrence TS and Ben-Josef E. Full-dose gemcitabine and concurrent radiotherapy for unresectable pancreatic cancer. Int J Radiat Oncol Biol Phys. 2007; 68(3): 801-808.

5. Loehrer PJ Sr, Feng Y, Cardenes H, Wagner L, Brell JM, Cella D, Flynn P, Ramanathan RK, Crane CH, Alberts SR and Benson $\mathrm{AB}$ 3rd. Gemcitabine alone versus gemcitabine plus radiotherapy in patients with locally advanced pancreatic cancer: an Eastern Cooperative Oncology Group trial. J Clin Oncol. 2011; 29(31): 4105-4112.

6. Chan A, Diamandis EP and Blasutig IM. Strategies for discovering novel pancreatic cancer biomarkers. J Proteomics. 2013; 81: 126-134.

7. Fong ZV and Winter JM. Biomarkers in pancreatic cancer: diagnostic, prognostic, and predictive. Cancer J. 2012; 18(6): 530-538.

8. Berger AC, Garcia M Jr, Hoffman JP, Regine WF, Abrams RA, Safran H, Konski A, Benson AB 3rd, MacDonald J and Willett CG. Postresection CA 19-9 predicts overall survival in patients with pancreatic cancer treated with adjuvant chemoradiation: a prospective validation by RTOG 9704 . J Clin Oncol. 2008; 26(36): 5918-5922.

9. Goonetilleke KS and Siriwardena AK. Systematic review of carbohydrate antigen (CA 19-9) as a biochemical marker in the diagnosis of pancreatic cancer. Eur J Surg Oncol. 2007; 33(3): 266-270.

10. Yamamoto M, Yoshinaga K, Matsuyama A, Tsutsui S and Ishida T. CEA/CA72-4 levels in peritoneal lavage fluid are predictive factors in patients with gastric carcinoma. $\mathrm{J}$ Cancer Res Clin Oncol. 2014; 140(4): 607-612.

11. Lenhard MS, Nehring S, Nagel D, Mayr D, Kirschenhofer A, Hertlein L, Friese K, Stieber P and Burges A. Predictive value of CA 125 and CA 72-4 in ovarian borderline tumors. Clin Chem Lab Med. 2009; 47(5): 537-542.

12. Louhimo J, Alfthan H, Stenman UH and Haglund C. Serum
HCG beta and CA 72-4 are stronger prognostic factors than CEA, CA 19-9 and CA 242 in pancreatic cancer. Oncology. 2004; 66(2): 126-131.

13. Jiexian J, Xiaoqin X, Lili D, Baoguo T, Ting S, Xianwen $\mathrm{Z}$ and Cunzhi H. Clinical assessment and prognostic evaluation of tumor markers in patients with gastric cancer. Int J Biol Markers. 2013; 28(2): 192-200.

14. Jiang XT, Tao HQ and Zou SC. Detection of serum tumor markers in the diagnosis and treatment of patients with pancreatic cancer. Hepatobiliary Pancreat Dis Int. 2004; 3(3): 464-468.

15. Wang $Z$ and Tian YP. Clinical value of serum tumor markers CA19-9, CA125 and CA72-4 in the diagnosis of pancreatic carcinoma. Mol Clin Oncol. 2014; 2(2): 265-268.

16. Shimada H, Noie T, Ohashi M, Oba K and Takahashi Y. Clinical significance of serum tumor markers for gastric cancer: a systematic review of literature by the Task Force of the Japanese Gastric Cancer Association. Gastric Cancer. 2014; 17(1): 26-33.

17. Szekanecz E, Szucs G, Szekanecz Z, Tarr T, AntalSzalmás P, Szamosi S, Szántó J and Kiss E. Tumorassociated antigens in systemic sclerosis and systemic lupus erythematosus: associations with organ manifestations, immunolaboratory markers and disease activity indices. J Autoimmun. 2008; 31(4): 372-376.

18. Xiong GP, Zhang JX, Gu SP, Wu YB and Liu JF. Overexpression of ECM1 contributes to migration and invasion in cholangiocarcinoma cell. Neoplasma. 2012; 59(4): 409-15.

19. Fan H, Guo Z, Gao L, Zhang Y, Fan D, Ji G, Du B and Wei Q. Ultrasensitive electrochemical immunosensor for carbohydrate antigen 72-4 based on dual signal amplification strategy of nanoporous gold and polyaniline$\mathrm{Au}$ asymmetric multicomponent nanoparticles. Biosens Bioelectron. 2015; 64: 51-56.

20. Rindi G, Falconi M, Klersy C, Albarello L, Boninsegna L, Buchler MW, Capella C, Caplin M, Couvelard A, Doglioni C, Delle Fave G, Fischer L, Fusai G, de Herder WW, Jann H, Komminoth P, de Krijger RR, La Rosa S, Luong TV, Pape U, Perren A, Ruszniewski P, Scarpa A, Schmitt A,Solcia E and Wiedenmann B. TNM staging of neoplasms of the endocrine pancreas: results from a large international cohort study. J Natl Cancer Inst. 2012; 104(10): 764-777. 